\title{
Design optimization for vibration reduction of viscoelastic damped structures using genetic algorithms
}

\author{
Zhengchao Xie, W. Steve Shepard Jr.* and Keith A. Woodbury \\ The University of Alabama, Department of Mechanical Engineering, 290 Hardaway Hall, Box 870276, Tuscaloosa, \\ AL 35487, USA
}

Received 15 May 2006

Revised 29 May 2008

\begin{abstract}
Due to the large number of design variables that can be present in complex systems incorporating visco-elastic damping, this work examines the application of genetic algorithms in optimizing the response of these structures. To demonstrate the applicability of genetic algorithms (GAs), the approach is applied to a simple viscoelastically damped constrained-layer beam. To that end, a finite element model (FEM) derived by Zapfe, which was based on Rao's formulation, was used for a beam with constrained-layer damping. Then, a genetic algorithm is applied to simultaneously determine the thicknesses of the viscoelastic damping layer and the constraining layer that provide the best response. While the targeted response is ultimately at the discretion of the designer, a few different choices for the fitness function are shown along with their corresponding impact on the vibratory response. By integrating the FEM code within the GA routine, it is easier to include the frequency-dependence of both the shear modulus and the loss factors for the viscoelastic layer. Examples are provided to demonstrate the capabilities of the method. It is shown that while a multi-mode optimization target provides significant reductions, the response for that configuration is inferior to the response when only single-mode reduction is considered. The results also reveal that the optimum configuration has a lower response level than when a thick layer of damping material is used. By demonstrating the applicability of GA for a simple beam structure, the approach can be extended to more complex damped structures.
\end{abstract}

Keywords: Genetic algorithms, viscoelastic, constrained layer damping, optimization

\section{Introduction}

For many engineering structures, such as beam and plate-like structures, it is often desired to reduce the vibration levels. One vibration reduction technique involves the application of constrained-layer damping, which incorporates the use of a viscoelastic material sandwiched between the primary structure and a constraining layer. In transverse vibration, the viscoelastic layer in this composite structure will strain in shear with hysteresis effects such that damping occurs. Ross et al. [1] proposed the first damping model for constrained layer damping. Their model, which assumes damping is only caused by shear deformation of the damping layer, is widely accepted for many applications and will be referred to here as the RUK model. Mead and Markus [2] derived a high-order partial differential equation for the viscoelastic-damped beam under different boundary conditions. That model can be considered an extension of the RUK model. Rao [3] derived a mass matrix and a stiffness matrix using Hamilton's principle, which can be used to solve the complex eigenvalue problem of constrained layer damping (CLD) structures. Rao's formulation was developed under four basic assumptions:

\footnotetext{
*Corresponding author. Tel.: +1 205348 0048; Fax: +1 205348 6419; E-mail: sshepard@eng.ua.edu.
} 


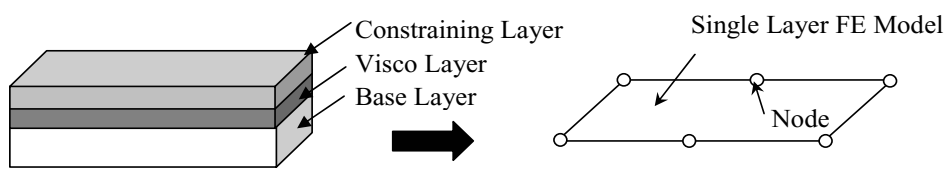

Fig. 1. The single-layer plate element used to represent a three layer structure.

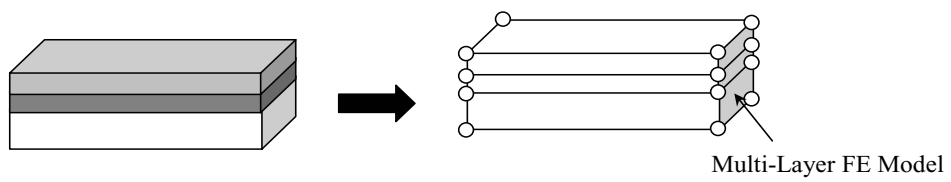

Fig. 2. Multi-layer solid element used to represent a three layer structure.

(1) The transverse displacements of all three layers are equal.

(2) The longitudinal displacement is linearly distributed across the thickness of each layer.

(3) All materials are isotropic and linear.

(4) There is no slip between the layers.

The sandwich beam in the Rao model has four longitudinal degrees of freedom, one for each of the outer and layer contacting surfaces, and one for the transverse displacement of the beam. This model includes the effect of longitudinal motion of each layer, which simultaneously allows for shear damping and extensional damping in the structure. These analytical models are very convenient for examining the impact of various design decisions on the response of the structure. Since all of the models mentioned thus far are for relatively simple beam structures, though, their direct application to more complex structures is quite limited.

In order to facilitate the consideration of more complex structures, numerous designs, and allow for the exchange of information among the various design entities, finite element methods (FEM) are often employed. There are two main methods used to model constrained-layer damped structures when using FEM: the single-layer-element method and multi-layer-element method, which are illustrated in Figs 1 and 2, respectively. The single-layer-element integrates the degrees of freedom (DOF) for all three layers. That is, the base layer, the viscoelastic layer, and the constraining layer are simultaneously modelled using one single-layer element. The multi-layer element, in which one element layer is used to represent each layer of the structure, more closely mimics the real structure. Obviously, the multi-layer-element method has more DOFs and thus will take more time in the mesh generation and computation stages. Although the multi-layer element can produce more precise results because it more closely mimics the actual structure, the increase in model complexity can sometimes be overwhelming, especially when the viscoelastic layer is very thin. The use of these two types of model formulations to analyze constrained-layer damping FEM applications will now be reviewed.

The approach used in this work will be based on a single-layer formulation. There are various approaches to modelling a three-layer structure with a single layer of finite elements. In one modelling approach, the theoretical RUK model is used with the single-layer method to construct a discretized single-layer-element that simultaneously represents all of the layers. Akanda et al. [4] used NASTRAN to set up a single-layer-element FEM shell element model of an automobile part. In that work, a subroutine was developed that would calculate the effective bending stiffness of a composite beam by using the equations from the RUK model. FEM modelling was then used to find the response of the structure under excitation. In that approach, the viscoelastic material is modelled with damping properties in whatever manner is provided by the modelling software. Although it is well known that the viscoelastic layer's shear modulus and loss factor typically vary with frequency, constant values are sometimes assumed for those properties. Rikards [5] assumed a constant loss factor of unity for the damping layer so that a standard eigenvalue method could be employed, as opposed to using the direct response method. Then a single-layer beam finite element was proposed to predict the loss factors and natural frequencies for the structure. In order to prove that the new element proposed by Rikards [5] performed better, Rikards compared the results for that element with other results that had been previously verified. Zapfe et al. [6] proposed a single-layer element which can be seen as the FEM implementation of Rao's [3] assumptions. Zapfe derived the stiffness and mass matrices for each layer first and 
then assembled those together to form the matrices for the combined three layers. The resulting mass and stiffness matrices can be used to obtain the eigenvalues, which contain the natural frequencies and modal loss factors of the sandwich beam.

Since it is often desired to obtain the best performance for a particular application, design optimization can often be a significant issue. Many optimization methods are available, such as calculus-based methods like the conjugate gradient method and differential correction. When these methods are not suitable, Genetic Algorithms (GAs) is a good choice because of its robustness. The GA is a method based on stochastic search, which requires only knowledge of the objective function and not any of its derivatives. GAs use natural evolutionary principles to obtain offspring that provide characteristics that are better than those of the parents. The optimization results are typically at or close to the global optimum. Some GA optimization methods have been applied to the constrained-layer damping problem. Trindade et al. [7] constructed a coupled FEM model for both active and passive damping, in which the core layer acts as passive damping and the piezoelectric layers act on the two outer layers. This model was combined with GAs to obtain an optimum configuration under hybrid (active and passive) damping control. Zheng et al. [8] used the assumptions of the RUK model in order to derive the governing equations for a constrained damping patch, which is made up of part of the constrained (i.e. part of the visco) layer on the beam. These equations were then used with GAs to minimize the vibration energy over a certain frequency range. Note that reference [8] considered the loss factor to be constant despite the fact that this property usually varies with frequency.

The purpose of this work is to reconsider the structure optimization problem for visco-elastically damped beams via the use of GAs. First, the frequency dependent properties of the viscoelastic layer are included in the singlelayer-element developed by Zapfe [6], which was based on Rao's model [3] as noted earlier. This model is then used to obtain the frequency response of a composite beam with a viscoelastic damping layer. A GA approach is then used with this model in order to find the thickness values for both the constrained layer and the constraining layer that provide an optimized structural response. The details of the specific optimization will be provided later. Basically, the GA relies on an evaluator to determine the "goodness" of a candidate solution and the FEM solver used herein offers some advantage. Specifically, compared with the assumption of the RUK model in which only shear damping is considered, the Zapfe element includes both shear damping and extensional damping. As such, this model is more accurate when extensional damping cannot be neglected. The precision of this FEM will be shown later in this work. In the following discussion, the mechanism of constrained layer damping and a brief background of GAs are provided. Then, a discussion of the current approach is provided along with results from a case study where the first goal is to simultaneously reduce the sum of the squares of the amplitudes for the first three modes. Then a comparison is made to the optimum solutions where the goal is to reduce the response of individual modes. The approach taken here is to demonstrate the applicability of GA to optimizing the simple beam problem. Once demonstrated for the cases and materials selected, the approach can easily be extended without much additional effort to optimize more complex damped structures that, unlike beam structures, don't have simple closed-form solutions.

\section{Damping mechanism in composite beams}

The damping layer of the sandwich beam is made up of a viscoelastic material with properties that can be described as

$$
E^{*}=e\left(1+j \eta_{e}\right)
$$

and

$$
G^{*}=G\left(1+j \eta_{g}\right)
$$

where $E^{*}$ is the complex Young's Modulus, $G^{*}$ is the complex shear modulus, $\eta_{e}$ is the Young's Modulus loss factor, and $\eta_{g}$ is the shear modulus loss factor. Note that $E$ is the real value of the Young's Modulus, and $G$ is the real value of the shear modulus. Although the loss factors $\eta_{e}$ and $\eta_{g}$ are each temperature- and frequency-dependent, these two values are often treated as equal to each other. Furthermore, these values are often simplified and treated as constants that do not vary with frequency. While the two parameters will be treated as equal in this work, the frequency dependence will be considered. 
In the Rao model, which is the basis for the formulation used here, the damping results from both the shear strain and extensional strain in the viscoelastic layer. Although temperature variations impact material properties and therefore should be considered in a detailed analysis, the temperature of the structure is assumed constant in this work. Note that the frequency-dependence of the properties has been considered. The goal of this work is to determine the thickness of the visco and constraining layers that will provide the structure with the lowest response amplitude given a particular excitation. There are several issues that must be considered prior to performing such an optimization.

Due to the frequency-dependence of the shear modulus in the viscoelastic layer, the complex-valued stiffness matrix $[K]$ in the vibration equation

$$
\left\{[K]-\omega^{2}[M]\right\}\{X\}=\{F\}
$$

varies with frequency. In this expression, the response for a given force vector $\{F\}$ is given by the vector $\{X\}$. Since the stiffness matrix varies with frequency, it is impossible to directly obtain the natural frequencies with the eigenvalue method. Only a direct method can be used to conveniently obtain the response and the resonance frequencies. That is, the equation

$$
\{X\}=\left\{[K]-\omega^{2}[M]\right\}^{-1}\{F\}
$$

must be solved directly and the peak values for $\{X\}$ used to estimate the resonance frequencies. In performing an optimization, the response of the beam at certain frequencies and under different configurations is used by the GA code to calculate the fitness. The fitness and objective function will be described below after the GA method is reviewed.

As noted above, the single-layer element proposed in [6] has been used and will be shown to perform well. Once the FEM code is formulated and the dynamic response is found, the GA code then searches for the optimal results. Numerical examples are provided below.

\section{Optimization via Genetic Algorithms (GAs)}

GAs are not new to the field of mechanical design. In 1975, Holland published the book 'Adaptation in Natural and Artificial System' [9], which systematically described the topic of GAs as applied to biological research. Since that time, GAs have been widely used in many fields such as structure optimization. In general, GAs use stochastic search and natural evolutionary principles as the basis for optimization. The algorithms first create a population of "individuals", which are typically binary strings coded to represent the parameters of the problem. The best elements of the population are then selected for various operations, which will be described below. The goal of each step in the algorithm is to obtain a new generation, referred to as offspring, which is closer to the optimal solution than the parent generation. This basic approach is illustrated in Fig. 3. Details associated with the steps in this optimization process will be discussed later.

In this work, the thickness of the constrained (i.e. viscoelastic) layer and the thickness of the constraining layer are the variables encoded into a binary string using a multi-parameter, mapped, fixed-point coding (Chapter 3 of Reference [10]). Each of the thicknesses is coded using 20 bits, so the total length of the string needed to describe the configuration of the sandwich beam is 40 bits. The population of each generation is made up of a specified number of such strings. The corresponding dynamic response of the beam, associated with each string is used to calculate the string's "goodness" or fitness. The fitness is used to ultimately determine the survivability of the particular string in the evolution. In one of the examples below the fitness is the reciprocal of the summation of the maximum amplitudes of the structural response at its first three resonant frequencies. The main steps in the GA are selection, crossover, and mutation. The population is propagated by first selecting two parent strings from the current generation and then mating these to produce two offspring. Selection of parents is biased toward those with higher fitness through a roulette wheel selection scheme. In the roulette wheel scheme, the binary string which has higher fitness will be apportioned a bigger part in the wheel and therefore will have a higher chance of being selected. The wheel spins twice consecutively to select two strings for mating. Mating is accomplished using a simple crossover operation in which the two parent strings exchange their bits from a randomly selected position, as illustrated in Fig. 4. After 


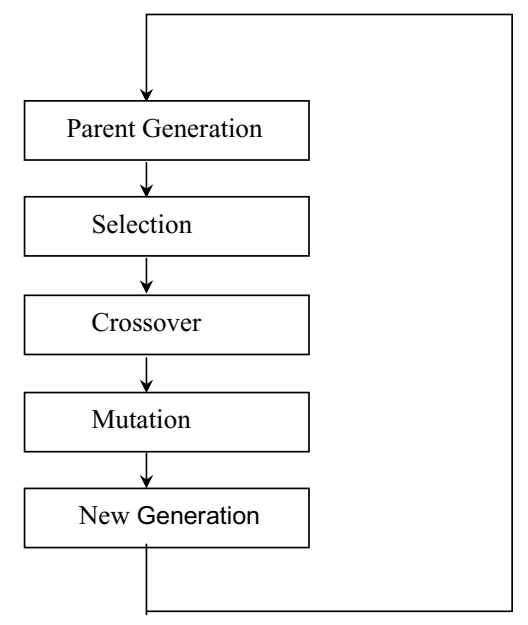

Fig. 3. Procedure for genetic algorithm.

\begin{tabular}{|c|c|c|}
\hline Parent 1: & 10011101 & $\begin{array}{llll}1 & 0 & 1 & 1\end{array}$ \\
\hline Parent 2: & 10001001 & $\begin{array}{llll}0 & 0 & 1 & 1\end{array}$ \\
\hline Offspring 1: & 100011101 & $\begin{array}{lllll}0 & 0 & 1 & 1\end{array}$ \\
\hline Offspring 2: & 10000010001 & $\uparrow_{\mathrm{Cros}}^{1011}$ \\
\hline
\end{tabular}

Fig. 4. Crossover operation in GAs.

$\begin{array}{llllllllllllll}\text { String before mutation: } & 1001001110 & 0 & 0 & 1 & 1 & 0 & 0 \\ \text { String after mutation: } & 100100101111 & 1 & 0 & 0 & 1 & \mathbf{0} & 0 & 0\end{array}$

Fig. 5. Mutation operation in GAs.

the new generation is created, mutation is applied to the strings in order to introduce variations into the population which are not present in the original generation. This operation selects one or several bits in the string and flips them, from 1 to 0 or 0 to 1, as illustrated in Fig. 5. Mutation plays a secondary role in the GA [10] by expanding the search space into regions not covered by the original populations, but should be used sparingly (perhaps with $1 \%$ to $5 \%$ probability). Although only two physical length measures are coded in this problem, any number of design parameters needed for a complex system design could be coded. For example, material cost could be a parameter varied in the optimization.

The entire flow of the GA code is shown back in Fig. 3. The initial population is created randomly within the range of allowed thickness for the constrained and constraining layers. The fitness of each string is then calculated in the FEM code. Selection, crossover, and mutation are then operated on this parent generation in order to obtain a new generation. Because of the evolution, the maximum fitness of the offspring generations should be better than the ancestor generations in the long run. The cycle continues until a specified number of generations have been created. The string with the maximum fitness of the last generation is the returned best optimal configuration for the sandwich beam. In this work, these GA operations are coded in Matlab, and the FEM code is based on the single-layer element given in Reference [6]. Note that here a beam element is used while the element illustrated 
Table 1

Parameters for the verification under different cases from [5]

\begin{tabular}{lccccc}
\hline Parameters & CASE 1 & CASE 2 & CASE 3 & CASE 4 & CASE 5 \\
\hline Layer Young's Modulus & & & & & \\
$\mathrm{E}_{1}\left(\mathrm{~N} / \mathrm{mm}^{2}\right)$ & 45540 & 45540 & 45540 & 45540 & 45540 \\
$\mathrm{E}_{2}\left(\mathrm{~N} / \mathrm{mm}^{2}\right)$ & 15.9 & 145 & 290 & 7.25 & 72.5 \\
$\mathrm{E}_{3}\left(\mathrm{~N} / \mathrm{mm}^{2}\right)$ & 45540 & 45540 & 45540 & 45540 & 45540 \\
Layer Density & & & & & \\
$\rho_{1}\left(\mathrm{~g} / \mathrm{cm}^{3}\right)$ & 2.04 & 2.04 & 2.04 & 2.04 & 2.04 \\
$\rho_{2}\left(\mathrm{~g} / \mathrm{cm}^{3}\right)$ & 1.2 & 1.2 & 1.2 & 1.2 & 1.2 \\
$\rho_{3}\left(\mathrm{~g} / \mathrm{cm}^{3}\right)$ & 2.04 & 2.04 & 2.04 & 2.04 & 2.04 \\
Layer Height & & & & & \\
$\mathrm{H}_{1}(\mathrm{~mm})$ & 3 & 3 & 3 & 4.2 & 4.2 \\
$\mathrm{H}_{2}(\mathrm{~mm})$ & 3 & 3 & 3 & 0.6 & 0.6 \\
$\mathrm{H}_{3}(\mathrm{~mm})$ & 3 & 3 & 3 & 4.2 & 4.2 \\
Layer Poisson's Ratio & & & & & \\
$\nu_{1}$ & 0.33 & 0.33 & 0.33 & 0.33 & 0.33 \\
$\nu_{2}$ & 0.45 & 0.45 & 0.45 & 0.45 & 0.45 \\
$\nu_{3}$ & 0.33 & 0.33 & 0.33 & 0.33 & 0.33 \\
\hline
\end{tabular}

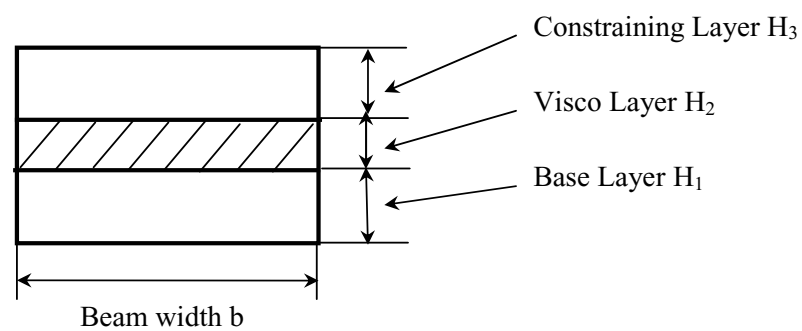

Fig. 6. Cross-section of the composite beam.

in Fig. 1 is a plate element. The FEM code reads the strings generated from the GA code and then calculates the dynamic response for each configuration. The dynamic response is then used in the embedded objective function in order to calculate the fitness for that particular solution. The particular objective functions considered for the optimization used herein are discussed below. This combination of GAs and FEM can also be used for optimizations in other, more complex, structures with appropriate modifications of the FEM and fitness functions.

\section{Approach verification}

Before using the single-layer FEM model in the GA optimization, this modelling approach should be validated. In the comparison provided by Rikards [5], a $0.27 \mathrm{~m}$ long sandwich beam simply-supported on both ends is considered, with the cross section shown in Fig. 6. Table 1 shows the five configurations of the sandwich beam from Reference [5] for comparison, with each configuration having a different Young's Modulus and a different thickness for each layer. Further details about each configuration can be found in Reference [5]. That work showed a good match between the results with Rikards' element and previous results. Consequently, Rikards results can be taken as accurate and used for validating the finite element formulation in the present work.

Table 2 shows a comparison between Rikards's and the FEM approach used in this work under the same conditions listed in Table 1. Clearly, the new element matches previous results well, especially when predicting the loss factors. Consequently, the performance of the current FEM is acceptable and the FEM code formulated here can be used to obtain the structure's dynamic response and used with the GA to optimize the response of the structure. In this work the beam is divided into 9 equal elements, which is more than enough to make the FEM computation converge. 
Table 2

Comparison of Rikards solution [5] to FEM results from this work

\begin{tabular}{|c|c|c|c|c|c|c|c|}
\hline \multirow[t]{2}{*}{ Case } & \multirow[t]{2}{*}{ Mode } & \multicolumn{3}{|c|}{ Loss Factor } & \multicolumn{3}{|c|}{ Frequency $(\mathrm{rad} / \mathrm{s})$} \\
\hline & & $\begin{array}{c}\text { Rikards } \\
\text { analytical } \\
\text { results }\end{array}$ & $\begin{array}{c}\text { Current } \\
\text { FEM }\end{array}$ & $\%$ Diff & $\begin{array}{c}\text { Rikards } \\
\text { analytical } \\
\text { results }\end{array}$ & $\begin{array}{c}\text { Current } \\
\text { FEM }\end{array}$ & $\%$ Diff \\
\hline \multirow[t]{3}{*}{1} & 1 & 0.50 & 0.496 & 0.8 & 878 & 928 & 5.7 \\
\hline & 2 & 0.34 & 0.335 & 1.5 & 2458 & 2536 & 3.2 \\
\hline & 3 & 0.20 & 0.195 & 2.5 & 4927 & 5041 & 2.3 \\
\hline \multirow[t]{3}{*}{2} & 1 & 0.20 & 0.196 & 2.0 & 1538 & 1554 & 1.0 \\
\hline & 2 & 0.43 & 0.431 & 0.2 & 4549 & 4758 & 4.6 \\
\hline & 3 & 0.50 & 0.494 & 1.2 & 7929 & 8419 & 6.2 \\
\hline \multirow[t]{3}{*}{3} & 1 & 0.11 & 0.112 & 1.8 & 1643 & 1650 & 0.4 \\
\hline & 2 & 0.31 & 0.313 & 1.0 & 5456 & 5601 & 2.7 \\
\hline & 3 & 0.45 & 0.448 & 0.4 & 9877 & 10391 & 5.2 \\
\hline \multirow[t]{3}{*}{4} & 1 & 0.32 & 0.321 & 0.3 & 1106 & 1134 & 2.5 \\
\hline & 2 & 0.20 & 0.202 & 1.0 & 3481 & 3533 & 1.5 \\
\hline & 3 & 0.11 & 0.111 & 0.9 & 7300 & 7419 & 1.6 \\
\hline \multirow[t]{3}{*}{5} & 1 & 0.10 & 0.104 & 4.0 & 1581 & 1586 & 0.3 \\
\hline & 2 & 0.26 & 0.26 & 0 & 5357 & 5456 & 1.8 \\
\hline & 3 & 0.32 & 0.317 & 0.9 & 10187 & 10492 & 3.0 \\
\hline
\end{tabular}

Table 3

Parameters for structure used in optimization

\begin{tabular}{llll}
\hline Properties & Layer 1 & Layer 2 & Layer 3 \\
\hline Density $\left(\mathrm{kg} / \mathrm{m}^{3}\right)$ & 2040 & 1200 & 2040 \\
Young's modulus & $45.54 \mathrm{e} 9$ & Frequency dependent & $45.54 \mathrm{e} 9$ \\
Shear modulus & N/A & Frequency dependent & N/A \\
Thickness(m) & 0.0042 & For optimization & For optimization \\
Length $(\mathrm{m})$ & 0.27 & 0.27 & 0.27 \\
Width $(\mathrm{m})$ & 0.015 & 0.015 & 0.015 \\
\hline
\end{tabular}

\section{Numerical study and optimization}

In order to examine the capabilities of the code, one can consider a sandwich beam simply supported at both ends with parameters as given in Table 3. For the GA parameters, the population size is 40 with single point crossover. Furthermore, forty bits are allocated for strings which represent the two design variables with twenty bits for each. The mutation rate is set as the reciprocal of the string length, which is 0.025 . For this study, the vibration amplitude of the beam due to a force that has unit amplitude in the frequency domain will be minimized. Consequently, the response will be presented in the frequency domain. While the structural response is minimized here for convenience, a designer may have stricter requirements that dictate minimizing some other response variable. So long as the fitness function is appropriately defined, any desired approach can be used. A schematic of the beam showing the location of the force is given in Fig. 7. The circles in the figure are used to denote the locations of the FE nodes on the beam.

In order to model the frequency dependence of the viscoelastic material, Lifshitz et al. [11] used two mathematical expressions that can be duplicated in this work. According to that work, the shear modulus is given by

$$
G=1.007 * 10^{-3} f+1.386 M P a,
$$

where $f$ is the frequency $(\mathrm{Hz})$. A plot of this expression is shown in Fig. 8. Note that the shear modulus monotonically increases throughout the frequency range considered. Correspondingly, the frequency-dependent loss factor is given by

$$
\eta=1.608 * 10^{-4} f+0.256 M P a .
$$

The loss factor is plotted in Fig. 9 for reference purposes. 


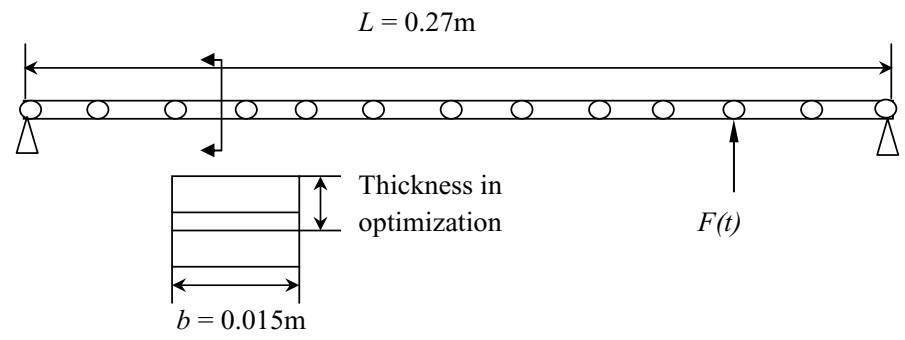

Fig. 7. Schematic of sandwich beam finite element model for the numerical study.

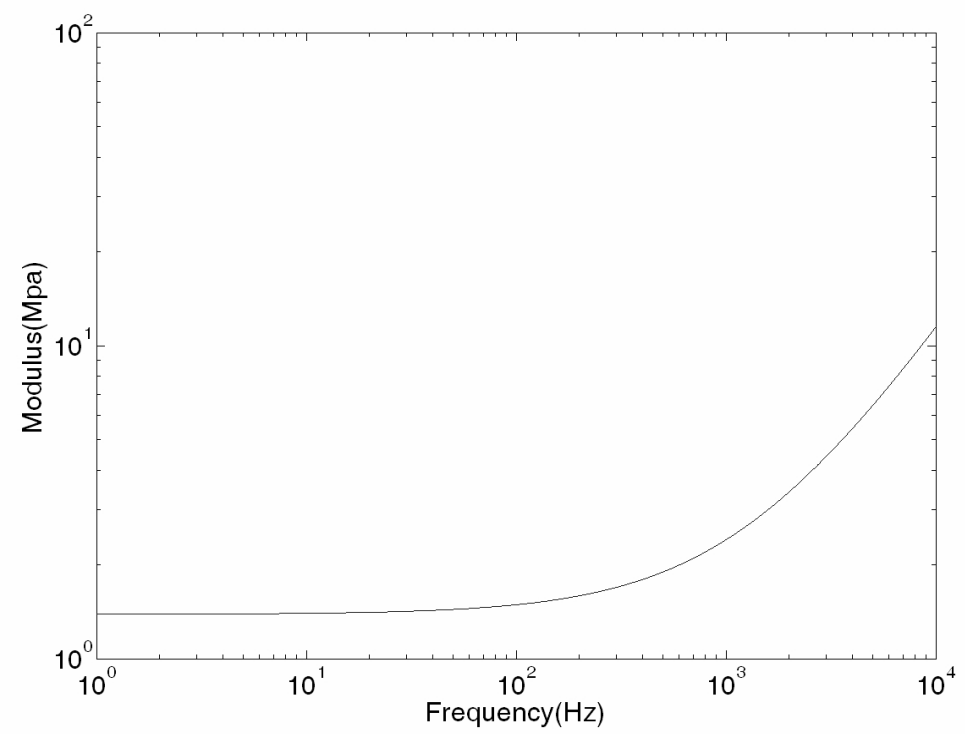

Fig. 8. The frequency-dependent amplitude of shear modulus.

Depending on the goals of the design, one can specify different objective functions for use in the GA optimization. Nakra [12] tried to maximize the system loss factor for certain modes. Zheng et al. [8] set minimization of the vibration energy as the objective. Because the response at the resonant frequencies is typically the most harmful, the initial objective function used here involves minimizing the overall response amplitude that would result from the simultaneous excitation of the first three modes each at their respective resonance frequency. For this case, the fitness of each population member is defined as $F S=\frac{1}{R_{1}^{2}+R_{2}^{2}+R_{3}^{2}}$ so that $\left(R_{1}^{2}+R_{2}^{2}+R_{3}^{2}\right)$ can be minimized when the GA finds the configuration of maximum fitness. Here, $R_{1}, R_{2}$ and $R_{3}$ are the peak responses of each population member for the first three resonant frequencies, respectively, at the location where the force is applied. Using squared values of the peak responses can increase the difference of fitness between the population members of each generation and this is beneficial for GAs optimization [10], though not required. As noted earlier, there are two design variables: 1) the thickness of constrained layer $H_{2}$ and 2) the thickness of constraining layer $H_{3}$, as illustrated in Fig. 7. In order to make the optimization practical, it is also necessary to specify limits on the thickness of the two layers. For this problem, it will be specified that the thickness for these two layers must be kept within a predefined practical range, which can be expressed as

$$
H_{2 \min }<H_{2}<H_{2 \max } \text { and } H_{3 \min }<H_{3}<H_{3 \max },
$$

where the maximum and minimum values of both layers are $H_{2 \max }=H_{3 \max }=0.002 \mathrm{~m}$ and $H_{2 \min }=H_{3 \min }=$ $0.0001 \mathrm{~m}$, respectively. The decoded values of the binary strings will be mapped into the range between the upper and lower limits automatically. 


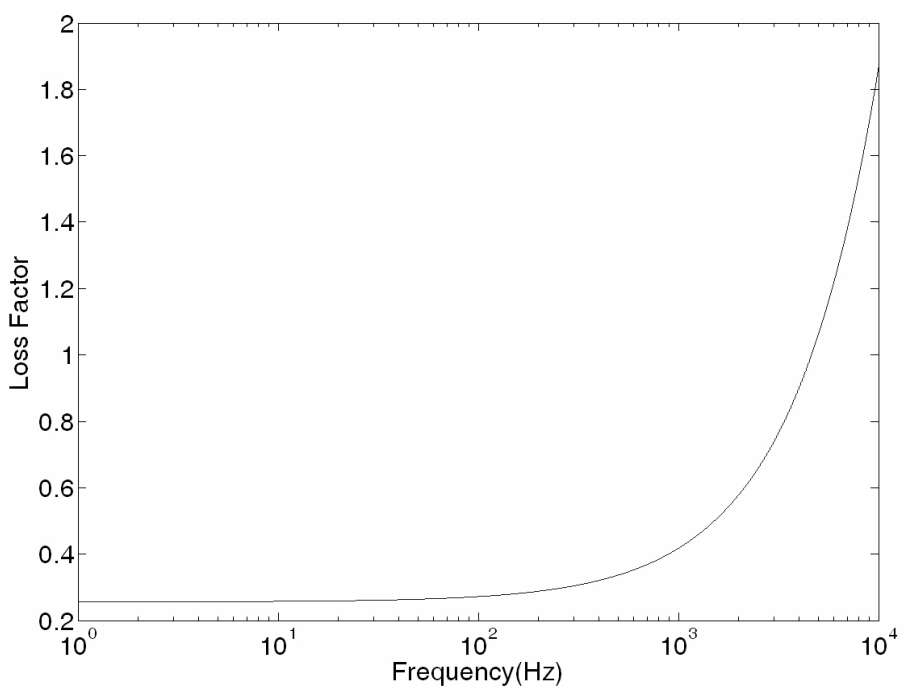

Fig. 9. The frequency-dependent loss factor.

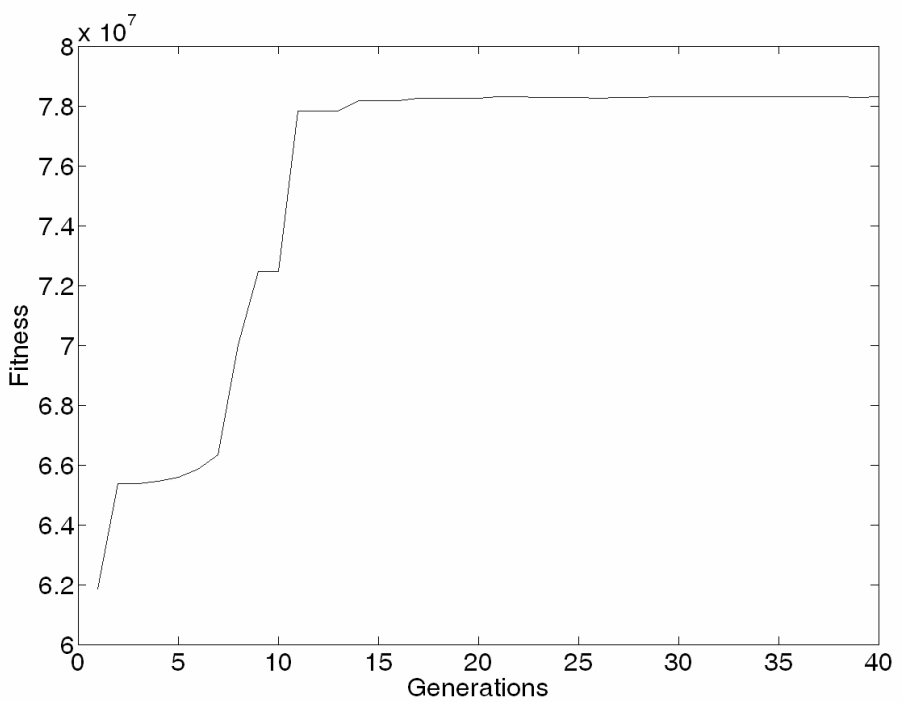

Fig. 10. Evolution of the best-so-far fitness vs. generation, during the overall optimization.

By using the above objective function and these layer thickness limits, the GA code will find the two layer thicknesses within those limits that provide a maximum value for FS. This case, where the first three resonances are considered simultaneously, will be referred to as the overall optimum case.

With the above settings, the GA and the FEM work together effectively such that the population appears to converge after the evolution of about sixteen generations, as shown in Fig. 10. The overall optimization, in which 40 generations are evaluated, takes several hours running on a $3.2 \mathrm{GHz}$ PC. If a larger population size and more generations are used, the optimization can take correspondingly longer. Given that calculus-based methods do not apply in these cases, though, time many not be such an issue.

Figure 11 shows the response for the overall optimum configuration. In this configuration, the resulting thicknesses for the layers are $0.0042 \mathrm{~m}, 0.00023 \mathrm{~m}$ and $0.002 \mathrm{~m}$ for the base layer, damping layer and constraining layer, 


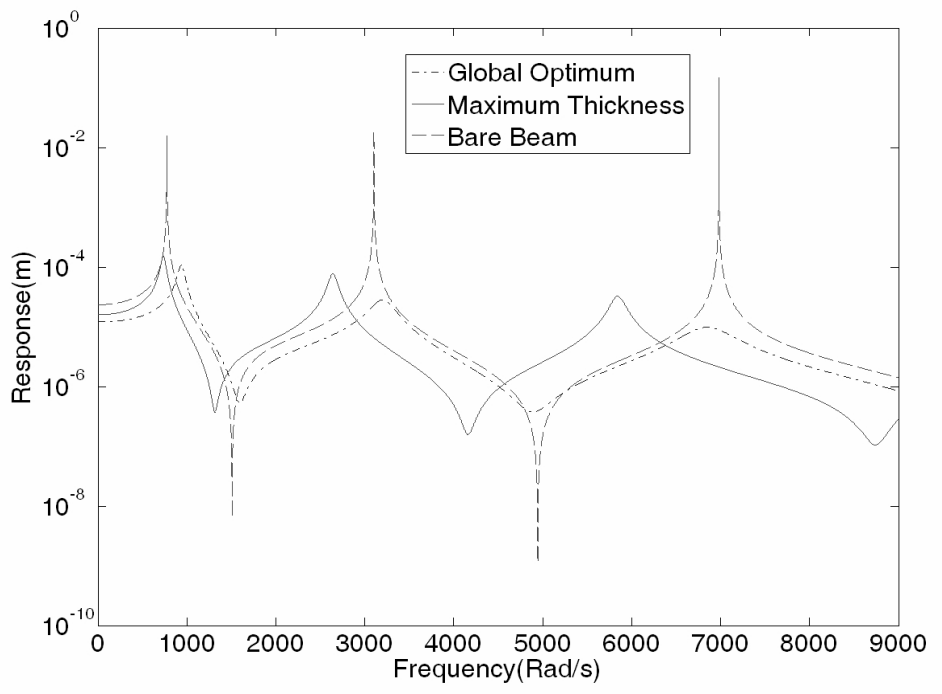

Fig. 11. Comparsion between the bare beam, the overall optimum configuration and the configuration with maximum thickness of damping layer and constraining layer.

respectively. The response for a bare beam with a thickness of $0.0042 \mathrm{~m}$ is also shown for comparison purposes. The response for a damped beam having layer thicknesses that are the maximum allowed by the configuration limits (i.e. $H_{2}=H_{2 \max }$ and $H_{3}=H_{3 \max }$ ) is also shown. Not surprisingly, the overall optimum configuration performs better than the maximum thickness configuration. Clearly, the response for both damped configurations is much lower than when using no damping.

In order to further examine the effectiveness of the overall optimum configuration, the three optimum configurations for the maximum suppression of the individual peak responses for the first, second and third resonances are also found. The location of response for these three cases is at the same as for the overall optimization case, at the location of the force. For each of these individual cases, the fitness can be written as $F S_{j}=\frac{1}{R_{j}}$, where the response amplitude $R_{j}$ is for the $j^{\text {th }}$ mode. Unlike the overall optimum, these optimum configurations provide the absolute lowest response possible for an individual resonance within the geometric limits given above. These three optimum configurations are referred to as the local optimum configurations. The response for these three configurations will each be compared with the overall optimum configuration results in order to see how the configuration and resulting response will differ given a different objective.

Figures 12 through 14 show the response for the optimum configurations when the objective is to minimize the response from individual modes. The layer thicknesses for each configuration are provided in the figure captions. The overall optimum is close to the first mode optimum. The reason for the small difference between the overall optimum and the local optimum of the first mode is the trade-off between the first mode optimum and optimums of the second and the third mode under the overall objective function. Because the first mode has a larger displacement amplitude for the case being considered, utilizing a sum of squared displacements for the first three modes results in the first mode dominating. For comparison purposes, the response shown in Fig. 11 for the overall optimum configuration is repeated in each of these figures. The response for using the maximum allowed thicknesses is also shown, again repeated from Fig. 11. In these three figures, it can be seen that the response is improved when optimizing for the response of the individual mode. That is, there is a slight trade-off in increased response when the objective is an overall reduction of the first three modes. This result is not surprising given the additional requirement of suppressing two additional modes. Obviously, each of the three optimum configurations performs better than the maximum thickness configuration. Depending on the goal of the design, one of the optimized solutions is preferable.

Upon further comparison of Figs 12, 13, and 14, it can be seen that the first mode of the damped beam has a higher response than the response for the other two modes. Since it is common to excite the first resonant frequency, the optimization of the first mode is important and may be more important than the alternative selection of the 


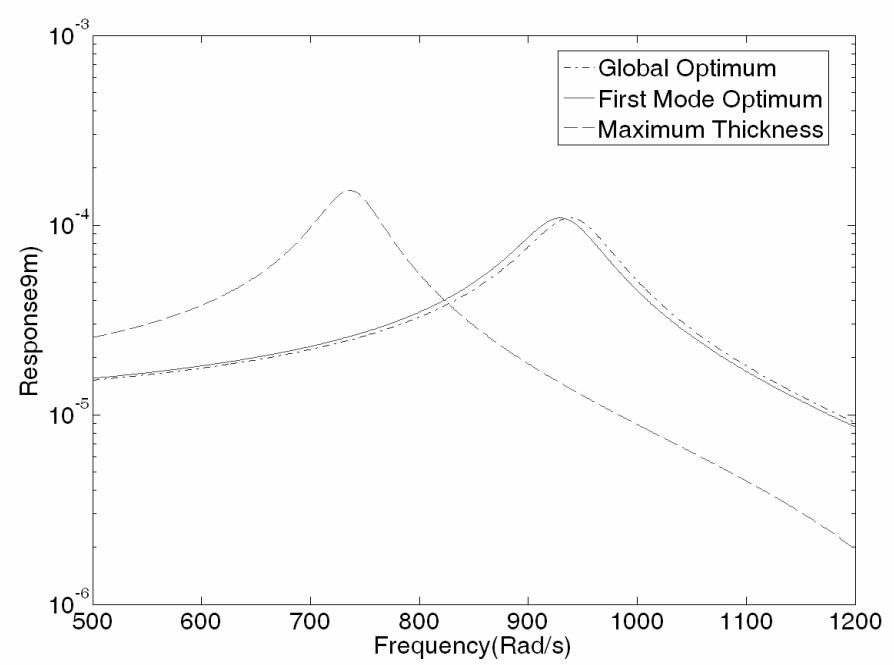

Fig. 12. Comparsion between the local optimum configuration for the first mode, the overall optimum configuration and the configuration with maximum thickness of damping layer and constraining layer. The visco and constraining layer thicknesses for the local optimum are $0.00025 \mathrm{~m}$ and $0.002 \mathrm{~m}$, and for maximum thickness configuration are $0.002 \mathrm{~m}$ and $0.002 \mathrm{~m}$, respectively.

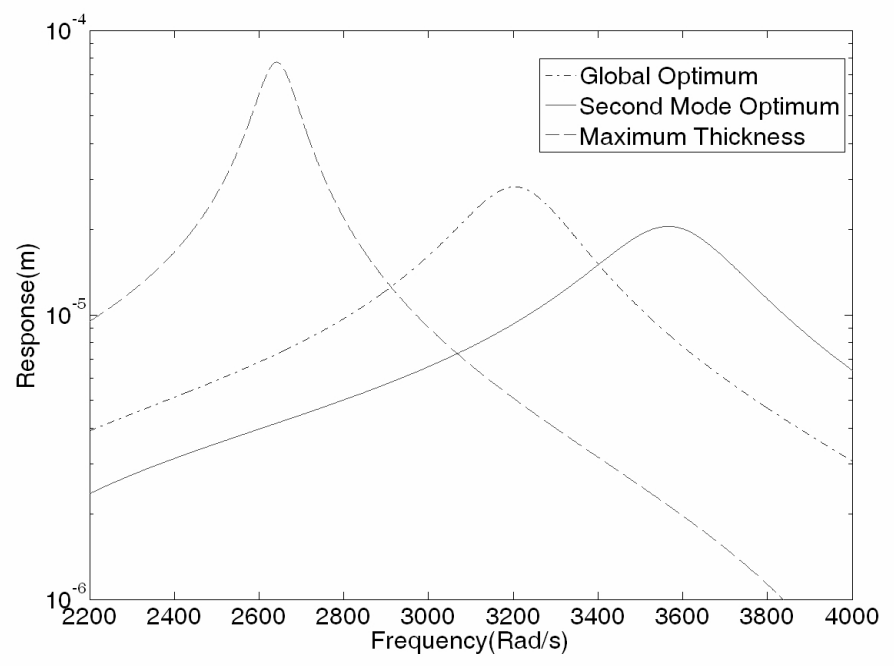

Fig. 13. Comparsion between the local optimum configuration in the second mode, the overall optimum configuration and the configuration with maximum thickness of damping layer and constraining layer. The visco and constraining layer thicknesses for the local optimum are $0.0001 \mathrm{~m}$ and $0.002 \mathrm{~m}$, and for maximum thickness configuration are $0.002 \mathrm{~m}$ and $0.002 \mathrm{~m}$, respectively.

overall optimum. Nevertheless, the selection of the best optimum approach must be based on some knowledge of the excitation and the critical response characteristics.

\section{Conclusion}

In this work, Zapfe's element was used to construct a single-layer-model for use in a FEM code. This element was used to obtain the response for a beam with frequency-dependent constrained layer viscoelastic material. In order to find the geometric configurations that provided the best desired structural response, a GA was used to minimize the 


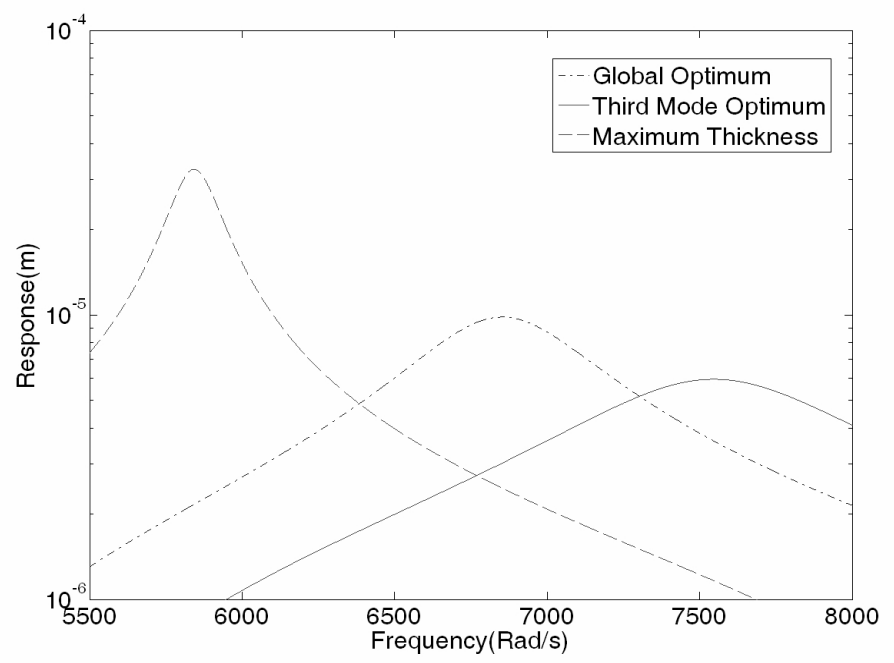

Fig. 14. Comparsion between the local optimum configuration in the third mode, the overall optimum configuration and the configuration with maximum thickness of damping layer and constraining layer. The visco and constraining layer thicknesses for the local optimum are $0.0001 \mathrm{~m}$ and $0.002 \mathrm{~m}$, and for maximum thickness configuration are $0.002 \mathrm{~m}$ and $0.002 \mathrm{~m}$, respectively.

response level of the sandwich beam in a frequency range that includes the first three modes. The GA method can obtain the response of the sandwich beam with a high efficiency, particularly when considering that calculus-based approaches may not be convenient or even applicable as the system complexity increases. The numerical examples showed that this approach has a good capability of optimizing a simple structure that includes constrained layer damping with both shear and extensional damping. In the cases examined, an overall objective function was set to minimize the simultaneous resonant response of the first three modes and the result shows that it can yield a satisfactory solution even when compared to the configurations for optimizing the response of individual modes.

\section{References}

[1] D. Ross, E.E. Ungar and E.M. Kerwin, Damping of Plate Flexural Vibrations by Means of Viscoelastic Laminae, Structural Damping, ASME Annual Meeting, 1959.

[2] D.J. Mead and S. Markus, The forced vibration of a three-layer, damped sandwich beam with arbitrary boundary conditions, Journal of Sound and Vibration 10 (1969), 163-175.

[3] D.K. Rao, Vibration of short sandwich beams, Journal of Sound and Vibration 52 (1977), 253-263.

[4] A. Akanda and G.M. Goetchius, Representation of constrained/unconstrained layer damping treatment in FEA/SEA vehicle system models: A simplified Approach, SAE (1999), 1999-01-168.

[5] R. Rikards, Finite element analysis of vibration and damping of laminated composites, Composite Structures 24 (1993), $193-204$.

[6] J.A. Zapfe and G.A. Lesieutre, A discrete layer beam finite element for the dynamic analysis of composite sandwich beams with integral damping layers, Computers \& Structures 70 (1999), 647-666.

[7] M.A. Trindade, Optimization of Sandwich/Multilayer Viscoelastic Structure for Vibration Damping, Proceeding of 20th International Conference on Offshore Mechanics and Arctic Engineering, 2001.

[8] H. Zheng and C. Cai, Optimization of partially constrained layer damping treatment for vibrational energy minimization of vibrating beams, Computers \& Structures 82 (2004), 2493-2507.

[9] J.H. Holland, Adaptation in Natural and Artificial System, The University of Michigan Press, Ann Arbor, 1975.

[10] D.E. Goldberg, Genetic Algorithms in Search, Optimization, and Machine Learning, Addison-Wesley Press, 1999.

[11] J.M. Lifshitz and M. Leibowitz, Optimal Sandwich Beam Design for Maximum Viscoelastic Damping, Int J Solids Structures 23 (1987), 1027-1034.

[12] B.C. Nakra, Vibration control in machines and structures using viscoelastic damping, Journal of Sound and Vibration 211 (1998), $449-465$. 

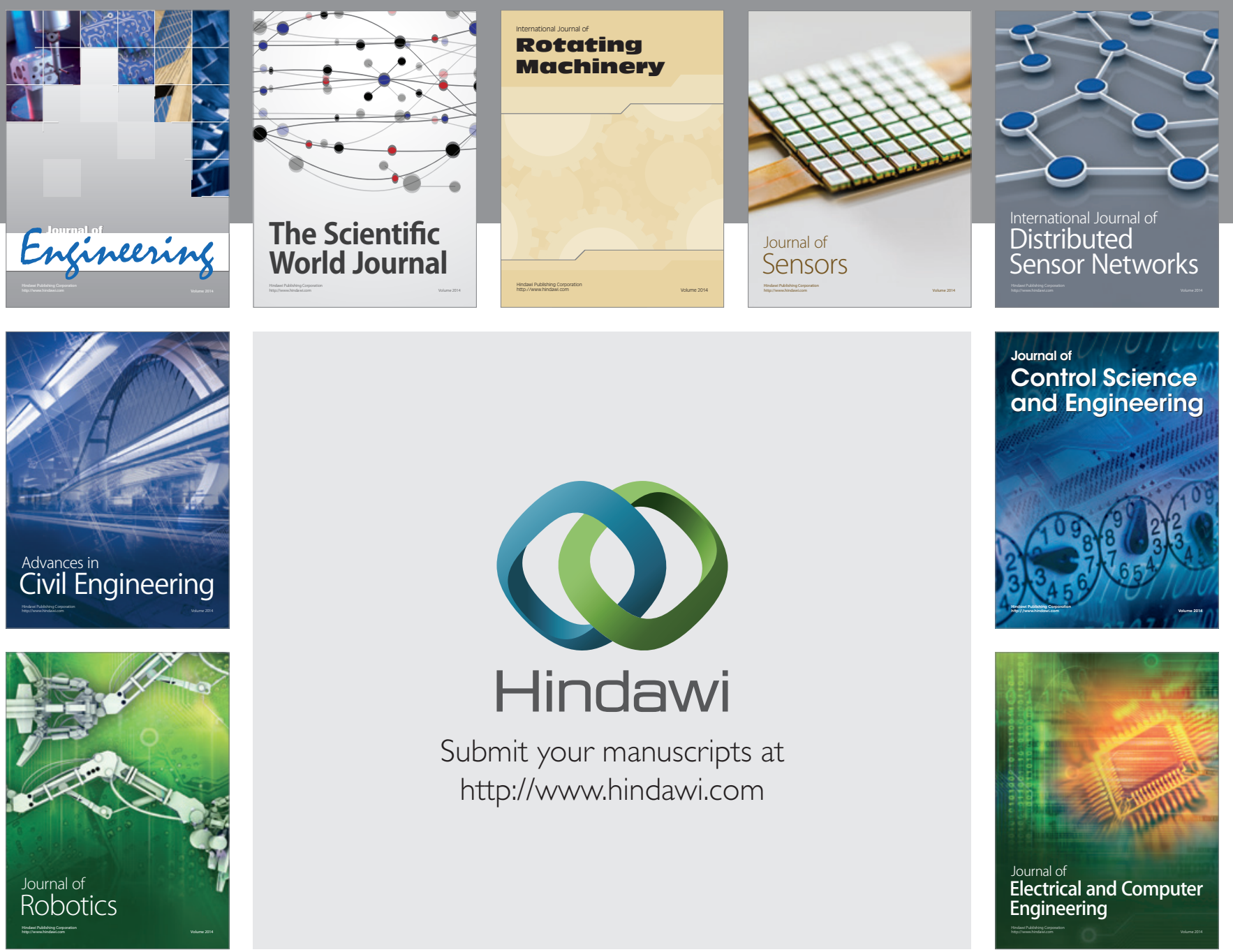

Submit your manuscripts at

http://www.hindawi.com
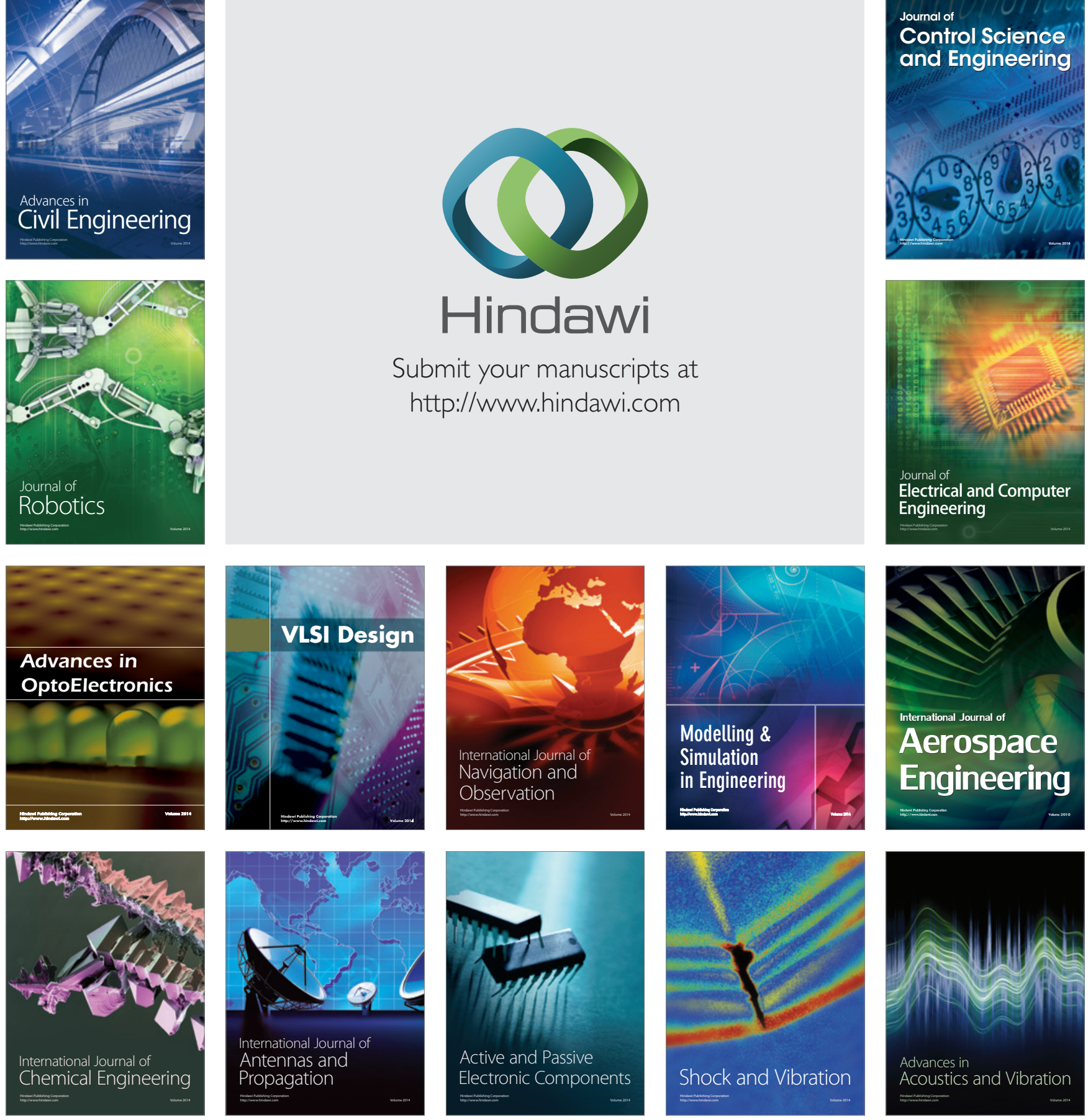